The Astrophysical Journal, 686:658-666, 2008 October 10

(C) 2008. The American Astronomical Society. All rights reserved. Printed in U.S.A.

\title{
HUBBLE SPACE TELESCOPE STIS OPTICAL TRANSIT TRANSMISSION SPECTRA OF THE HOT JUPITER HD 209458b
}

\author{
David K. Sing, ${ }^{1}$ A. Vidal-Madjar, ${ }^{1}$ J.-M. Désert, ${ }^{1}$ A. Lecavelier des Etangs, ${ }^{1}$ and G. Ballester ${ }^{2}$ \\ Received 2008 February 26; accepted 2008 May 6
}

\begin{abstract}
We present the transmission spectra of the hot Jupiter HD 209458b taken with the Space Telescope Imaging Spectrograph aboard the Hubble Space Telescope. Our analysis combines data at two resolutions and applies a complete pixel-by-pixel limb-darkening correction to fully reveal the spectral line shapes of atmospheric absorption features. Terrestrial-based $\mathrm{Na}$ I and $\mathrm{H}$ i contamination are identified that mask the strong exoplanetary absorption signature in the Na core, which we find reaches total absorption levels of $\sim 0.11 \%$ in a $4.4 \AA$ band. The Na spectral line profile is characterized by a wide absorption profile at the lowest absorption depths and a sharp transition to a narrow absorption profile at higher absorption values. The transmission spectra also show the presence of an additional absorber at $\sim 6250 \AA$, observed at both medium and low resolutions. We performed various limb-darkening tests, including using high-precision limb-darkening measurements of the Sun to characterize a general trend of ATLAS models to slightly overestimate the amount of limb darkening at all wavelengths, likely due to the limitations of the model's one-dimensional nature. We conclude that, despite these limitations, ATLAS models can still successfully model limb darkening in high signal-to-noise ratio transits of solar-type stars, like HD 209458, to a high level of precision over the entire optical regime $(3000-10000 \AA)$ at transit phases between second and third contact.
\end{abstract}

Subject headings: methods: data analysis — planetary systems — stars: individual (HD 209458)

\section{INTRODUCTION}

Transiting hot Jupiter planets offer a tremendous opportunity to study the characteristics of extrasolar planets. During a transit event, both the opaque body of the planet and its atmosphere block light from the parent star. A precise determination of the radius of the planet can be made from the total obscuration, while partial transmission of light through the exoplanet's atmosphere, along with its wavelength dependence, allows for the detection of composition and structure (Charbonneau et al. 2002; Ballester et al. 2007). In the middle of the visible spectrum, transits can probe atmospheric pressures from about 0.1 to $1 \times 10^{-6} \mathrm{bar}$, containing the troposphere (lower atmosphere), as well as the stratosphere and mesosphere (middle atmosphere). One exoplanet in particular, HD 209458b, has been ideal for these studies and holds the distinction of the first detection of an extrasolar planetary atmosphere (Charbonneau et al. 2002), as well as the discovery of an escaping atmosphere (Vidal-Madjar et al. 2003, 2004). These transit studies were performed with the Hubble Space Telescope (HST) using the Space Telescope Imaging Spectrograph (STIS).

Here we expand on earlier HST STIS analysis (Brown 2001; Charbonneau et al. 2002; Knutson et al. 2007; Ballester et al. 2007) in order to fully probe the line shape of atmospheric $\mathrm{Na}$ in HD 209458b and quantify other absorbers in the transmission spectrum. We use STIS data obtained during planetary transit at two spectral resolutions (low and medium). The two data sets are combined to extend the measurements over the entire optical regime, providing a way to simultaneously measure both the narrow core and wide line wing $\mathrm{Na}$ absorption. We describe these observations in $\S 2$, perform various limb-darkening tests in $\S 3$, present analysis of these data in $\S 4$, and discuss the results

\footnotetext{
${ }^{1}$ Institut d'Astrophysique de Paris, CNRS, Université Pierre et Marie Curie, 98 bis Boulevard Arago, F-75014 Paris, France; sing@iap.fr.

2 Lunar and Planetary Laboratory, University of Arizona, Sonett Space Science Building, Tucson, AZ 85721-0063.
}

in $\S 5$. From our resulting spectra, a detailed atmospheric model fit is given in Sing et al. (2008), which discusses condensation of sodium, as well as the implications of the atmospheric temperatures found. In addition, details of $\mathrm{H}_{2}$ Rayleigh scattering are presented in Lecavelier des Etangs et al. (2008), while Désert et al. (2008) explores absorption by atmospheric $\mathrm{TiO}$ and VO.

\section{OBSERVATIONS}

\subsection{Data Reduction}

The HST STIS G750M, G750L, and G430L observations of HD 209458b analyzed here are also detailed in Charbonneau et al. (2002), Ballester et al. (2007), Brown (2001), and Knutson et al. (2007). The G750M data consist of 684 spectra covering the wavelength range of 5518-6382 $\AA$ with a resolution $R$ of $\lambda / \Delta \lambda=5540(\sim 2$ pixels; $1.1 \AA)$ and taken with a wide $52^{\prime \prime} \times 2^{\prime \prime}$ slit to minimize slit light losses. This observing technique produces spectra which are photometrically accurate near the Poisson limit during the transit event. Four transits were observed, although the first suffered from a database error of the position of the spectrum and, as in Charbonneau et al. (2002), was not used for this analysis.

The data set was pipeline-reduced with the latest version of CALSTIS and cleaned for cosmic-ray detections before performing spectral extractions. The aperture extraction was done in IRAF software with a 30 pixel wide aperture, no background subtraction, and the optimal extraction algorithm of Horne (1986). This extraction technique produces spectra with $\sim 5 \%-6 \%$ more flux than simply summing over the cross-dispersion direction, as the stellar counts from the wings of the PSF can be added without additional noise.

The spectra were Doppler-corrected to the heliocentric rest frame, with velocity shifts on the order of 1 pixel. An additional subpixel shift in the dispersion direction was removed in each spectrum by cross-correlation against a template spectrum, which consisted of the mean pixel-shifted spectra. Such subpixel shifting is likely due to the jitter of the spacecraft, which changes the 


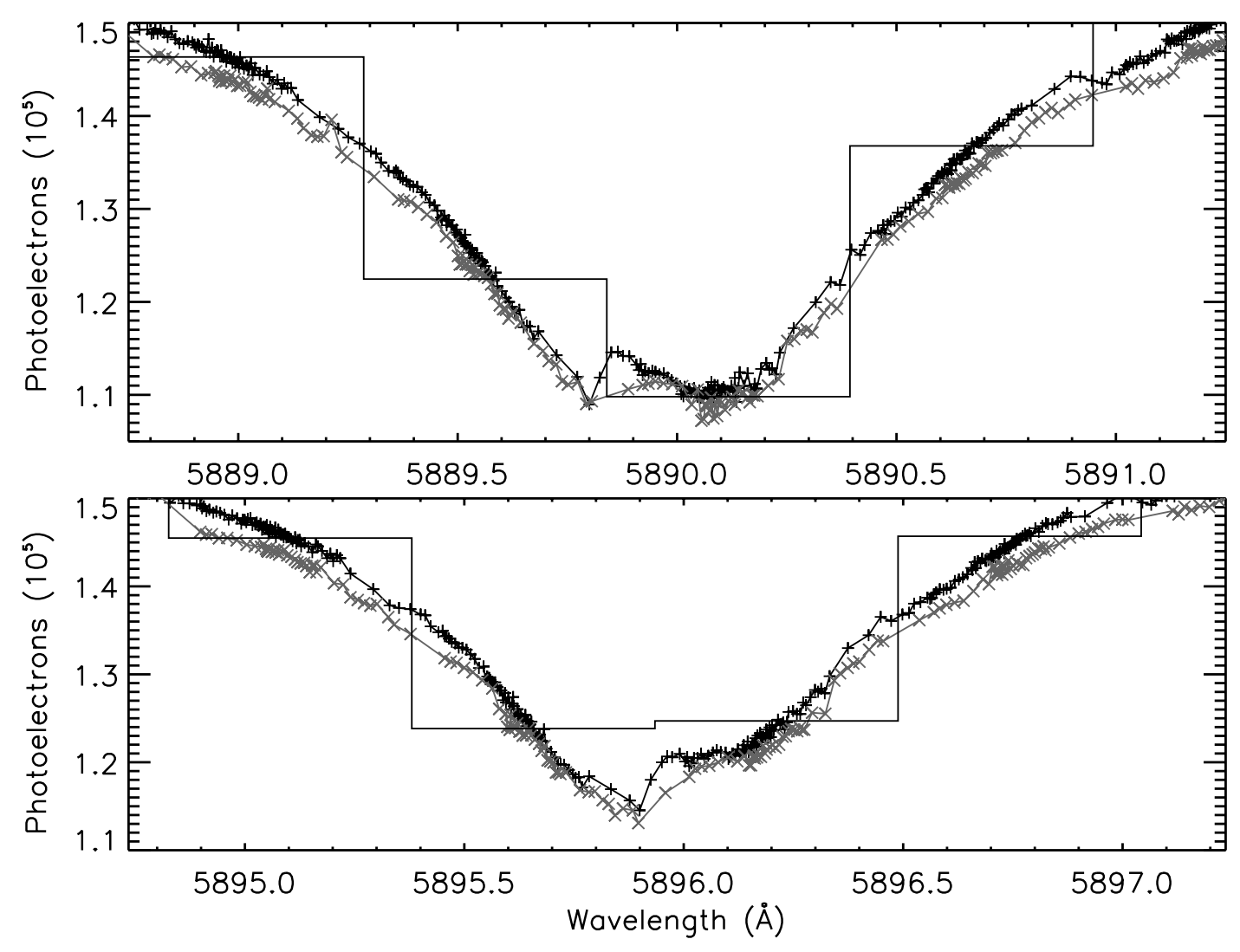

FIG. 1.-G750M spectra of the Na D2 (top) and D1 (bottom) line cores. Plotted are all of the out-of-transit spectra (black plus signs), as well as the in-transit spectra ( gray crosses). The uncertainty in counts for each point is dominated by photon noise (approximately \pm 400 photoelectrons) and is smaller than the plotted symbol size. The first out-of-transit spectrum in the series is plotted in histogram mode to show the size of each pixel, $0.554 \AA$. Plotted together, each individual spectrum's Doppler and pixel shifts (accurate to $\sim 0.1$ pixel) effectively add up to better sample the PSF of the stellar absorption line profile. The $\sim 1.5 \%$ difference in flux during transit can be seen in the flux difference between the two spectra. However, the region showing contamination from 5889.5 to $5889.7 \AA$ and from 5895.5 to $5895.8 \AA$ shows little to no difference in flux during transit. This region shows variations with the HST orbital period, which we attribute to terrestrial-based sodium contamination.

position of the star within the slit. The shifts are typically on the order of $\sim 0.1$ pixel within an orbit (the typical guiding accuracy of $H S T$ ) and approximately \pm 0.5 pixel from orbit to orbit. Several iterations of measuring pixel shifts, applying those shifts, and producing a new template were performed in order to create a reliable final template spectra for cross-correlation. With this method, we were able to align the spectra to $\sim 0.1$ pixel. With different Doppler and pixel shifts for each spectrum, the PSF of the stellar absorption line profiles can be reconstructed with a much greater sampling than can be obtained from an individual spectrum (see Fig. 1).

The extracted STIS spectra were then used to create a photometric time series covering the G750M bandpass by integrating the flux from each exposure. The resulting photometric light curve exhibits all the systematic instrumental effects noted in Brown (2001). As in both Brown (2001) and Charbonneau et al. (2002), we corrected for these systematic effects by discarding the first exposure of each orbit, discarding the first orbit of each visit, and applying flux corrections by removing a fourthorder polynomial fit to the photometric time series phased on the HST orbital period. The photometric corrections were then applied to the spectra themselves, such that different photometric bands could easily be produced.

The Na D wavelength region is also covered by HST STIS G750L observations, described by Knutson et al. (2007), although at much lower resolution. The G750L data set was also reduced, in the same manner as the G750M. The cross-correlation analysis, however, was only performed over the blue half of the data, such that our measured pixel shifts would be independent of the observed fringes.

\subsection{Telluric Contamination}

Telluric contamination can be seen in a photometric G750M time series of the Na core, where we see a large variation of $4 \%-5 \%$ over an $H S T$ orbit (see Fig. 2). This variation is observed to be correlated with the $H S T$ orbital phase, repeats in a similar manner within each orbit of a visit, and occurs in all three visits analyzed. The contamination occurs for both $\mathrm{Na}$ lines over the same radial velocities, approximately -5 to $-20 \mathrm{~km} \mathrm{~s}^{-1}$ from the $\mathrm{Na} \mathrm{D}$ line cores, and is consistent with the expected velocity of the Earth during the HST observations. Given the size of the variation, it is too large to be due to the known instrumental systematic effects, which only affect the light curves $\sim 0.1 \%$ during an orbit. In addition, other strong stellar lines such as Fe I do not show any signs of similar contamination, ruling out possible detector efficiency and count-rate-dependent issues as the cause of the observed line core variations. The contamination can also be seen directly in the G750M spectra (Fig. 1), observed as an $\sim 0.3 \AA$ region in the Na D cores with little to no flux drop during planetary transit, although the actual contaminating region could result from a smaller unresolved region. Absorption by interstellar Na could also be affecting the contaminated region, although its effect would be constant and should not vary with the $H S T$ orbital period. The G750L data set also shows telluric $\mathrm{Na} \mathrm{D}$ contamination, with $\mathrm{H} \alpha$ showing a similar effect as well. With lower resolution, the telluric signature is seen in photometric 


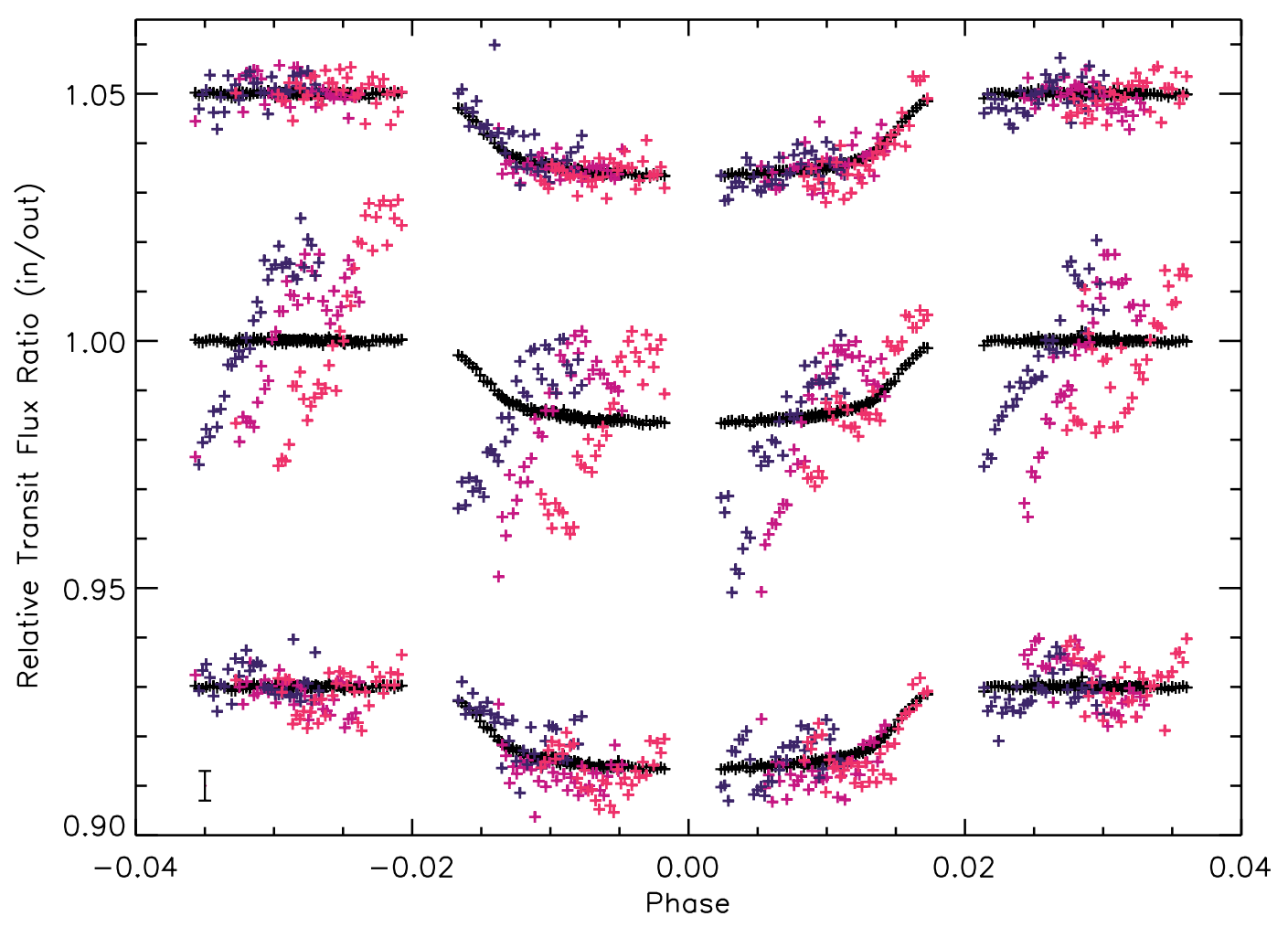

FIG. 2.-G750M photometry of the contaminated region. Plotted are photometric curves from the comparison region, which includes wavelengths between the Na lines

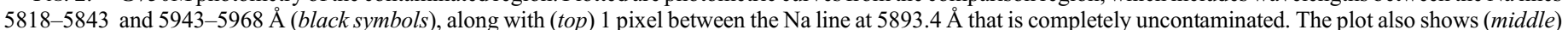
the same comparison region along with a highly contaminated pixel in the Na D1 line core at $5895.65 \AA$ and (bottom) a nearby D1 line-core pixel that is largely uncontaminated. A representative error bar for the single-pixel photometric light curves is also shown in the lower left-hand corner; the error for the comparison region is significantly smaller than the symbol size. The three colors ( purple, blue, and magenta) distinguish the three different $H S T$ visits, each consisting offive consecutive orbits (four orbits plotted). The telluric contaminated Na core can be seen as an $\sim 4 \%-5 \%$ change in flux which modulates on the $H S T$ orbital cycle, repeating each orbit.

time series measurements of the line cores with, as expected, a correspondingly lower amplitude than the medium-resolution data.

\subsection{Sodium Core Absorption Signature}

We performed a double differential photometric measurement, similar to that of Charbonneau et al. (2002), selecting 23 wavelength bands from $\sim 4$ to $100 \AA$ centered on the $\mathrm{Na} \mathrm{D}$ lines. In the original analysis, Charbonneau et al. (2002) used three different wavelength bands. We chose to use the sum of two bands, each centered on one component of the Na doublet for the two wavelength bands smaller than the Na D doublet separation. For each band, we integrated the spectral flux, producing photometric light curves. We then normalized each visit to their average out-of-transit flux. The two narrowest bands showed terrestrial contamination modulating on the HST orbital period, which we removed by fitting each visit of the HST phased out-of-transit flux with a fourth-order polynomial, applying the fit to the intransit orbits, as was done in a similar manner for the instrumental systematic effects. The photometric curves were then subtracted from a comparison band composed of the wavelength regions between 5818-5843 $\AA$ and 5943-5968 $\AA$, picked to be identical to the "wide" comparison band in Charbonneau et al. (2002). The relative planetary $\mathrm{Na}$ absorption during transit was then computed from the mean of the difference light curve over the in-transit phases between second and third contact, with its associated error computed from the variance of the data. Changing the width of the comparison bands in the same manner as Charbonneau et al. (2002) produced similar results for corresponding bands. Our resulting absorption profile (see Fig. 3) matched the results of Charbonneau et al. (2002) and, in addition, revealed the strong absorption from the $\mathrm{Na} \mathrm{D}$ line centers. When left uncorrected for terrestrial-based contamination, the two narrowest bands produced similar absorption values but with error bars large enough for the absorption to be consistent with a null detection.

A double differential spectroscopic measurement was also performed over the Na region. For the spectroscopic measurement, each spectra was first interpolated, using a cubic spline, onto a common wavelength scale. The spectral flux at each pixel was then normalized by the average out-of-transit flux and subtracted from the average normalized flux of the "wide" comparison region. The absorption at each wavelength during transit was then measured as performed for the photometric analysis, including telluric corrections for each pixel. The resulting differential spectra show two statistically significant positive absorption peaks at the $\mathrm{Na} \mathrm{D}$ lines with each having a large negative absorption core where the terrestrial contamination is largest (see Fig. 4). The telluric corrections on the $\mathrm{Na}$ lines have a modest effect on the absorption values outside the two largest contaminated pixels, as the telluric perturbation originates in a small subpixel region, with nearby pixels affected through the $\sim 2$ pixel instrument resolution. Excluding the two highly contaminated pixels effectively removes the majority of the contamination. In a 4 pixel bin, the average value of the two line cores is consistent with the $4.4 \AA$ band photometry measurement, indicating that both approaches to correcting the contamination give consistent values. In our final limb-darkened, corrected transmission spectra (see $\S 4.3$ ), we ultimately choose to apply the telluric corrections over the Na region while also leaving out the two highest 


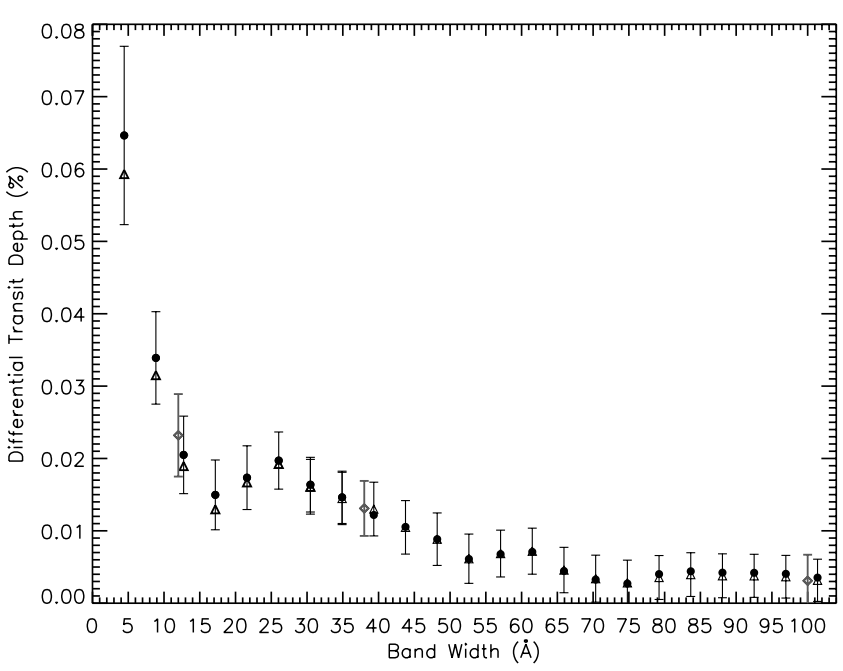

FIG. 3.-Double differential photometric Na core absorption. Plotted are the measured absorptions of various-size wavelength bands, each centered on the $\mathrm{Na} D$ lines, compared to that of a "wide" comparison region composed of the regions between 5818-5843 and 5943-5968 A (triangles). The limb-darkened corrected points (circles), with the associated $1 \sigma$ error bars, and the original three Charbonneau et al. (2002) measurements (gray diamonds) are also plotted. For each of our 23 wavelength bands, we integrated the spectral flux to produce transit light curves. These photometric light curves were then subtracted from the light curve of the "wide" comparison band. The atmospheric sodium absorption and associated error from each band were then measured by means of the difference light curve from the in-transit phases, between second and third contact. The two narrowest bands were corrected for telluric contamination and revealed a stronger $\mathrm{Na} \mathrm{D}$ core absorption.

contaminated pixels. Binning the differential spectra by 3 pixels $(1.66 \AA)$ shows that the Na D2 line is stronger than the absorption of the D1 line, with absorption values of $0.095 \% \pm 0.02 \%$ and $0.051 \% \pm 0.02 \%$, respectively, and an absorption ratio of $\mathrm{D} 2 / \mathrm{D} 1=1.9 \pm 0.9$. The differential photometric measurement includes these two pixels, as their effects are negligible at large bandwidths and correctable for the 4.4 and $8.9 \AA$ bands.

\subsection{Limb-darkening Corrections}

Correcting for limb-darkening effects allows a full transmission spectrum to be produced over the entire wavelength range of the STIS data. Similar to Ballester et al. (2007), we compute nonlinear limb-darkening coefficients to estimate and correct for the limb-darkening effects in the transit light curve. Although other limb-darkening laws could, in principle, be used (e.g., linear, quadratic, square root, or logarithmic), Claret (2000) found that the nonlinear law best represents the intensity distributions calculated from the one-dimensional plane-parallel atmospheric models used here.

We choose a Kurucz ATLAS stellar atmospheric model ${ }^{3}$ based on the model's limb-darkening performance, its performance versus other one-dimensional models, model availability, and development within the literature. Phoenix models (Claret 2000) were found to predict a stronger limb darkening, compared to ATLAS models, by a few percent in the photometric bands $B, V$, $R, I, J$, and $H$ but up to $40 \%$ weaker in the $U$ and $u^{\prime}$ bands. As the ATLAS models performed better compared to the Sun ( $\operatorname{see} \S 3.2)$, they were chosen over the Phoenix models for our study.

Most studies fitting transit light curves have chosen to either adopt an ATLAS stellar atmospheric model to account for limb-

\footnotetext{
${ }^{3}$ See http://kurucz.harvard.edu/stars/hd209458
}

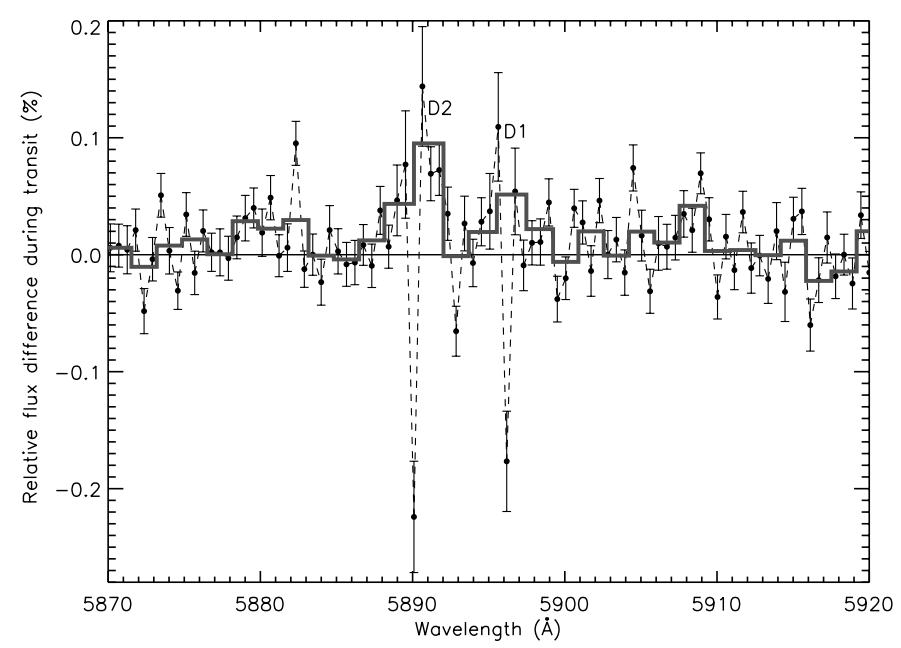

FIG. 4.-Double differential spectroscopic measurement. Plotted is the differential absorption at each wavelength in the Na region compared to the "wide" comparison band, along with the associated $1 \sigma$ error. Overplotted ( gray histogram) are the data binned over 3 pixels with the two large negative points, contaminated by terrestrial absorption, excluded in the bin. The $\mathrm{Na} \mathrm{D} 1$ and D2 doublet is resolved and seen as the two largest absorption peaks.

darkening effects or fit for limb-darkening parameters assuming a limb-darkening law. Knutson et al. (2007), who also used an ATLAS model for HD 209458, found similar planetary parameters when comparing the two methods, suggesting that modelbased limb-darkening fits can perform well. Knutson et al. (2007) chose to use the limb darkening calculated from the ATLAS models in their results, due to the more precise planetary parameters found and concerns that fitted limb-darkening coefficients could be affected by residual correlation in the light curve. Such concerns are valid, as we found that residual correlated "red" noise ultimately limits the maximum signal-to-noise ratio $(\mathrm{S} / \mathrm{N})$ achievable in this study (see $\S 4.2$ ). In addition, as our limbdarkening corrections can be on a pixel-by-pixel level, a light curve generated by any one particular pixel will not have sufficient $\mathrm{S} / \mathrm{N}$ to accurately fit for limb-darkening coefficients.

The four nonlinear coefficients were computed using intensity spectra from a Kurucz one-dimensional plane-parallel model atmosphere calculated at 20 different angles spread uniformly in $\mu$, fitting for the coefficients in the desired wavelength range. We calculated coefficients for the wavelength ranges used in the G750M differential measurements and each pixel of the G750M and G750L dispersions. Using the fit coefficients, we then used the theoretical transits of Mandel \& Agol (2002) to determine and correct for limb-darkening effects. The best-fit system parameters of Knutson et al. (2007) were used to calculate the impact parameter and planetary/star radius contrast. For the differential photometric measurement probing the $\mathrm{Na}$ line cores, designed by Charbonneau et al. (2002) to be largely independent of limb-darkening, all the corrections had a less than $1 \sigma$ effect, with corrections to our narrowest band only increasing the absorption by $0.005 \%$ (see Fig. 3).

\section{LIMB-DARKENING TESTS}

\subsection{Stellar Line versus Continuum Limb-darkening Strength Measurement}

When measuring $\mathrm{Na}$ in the G750M photometric bandpass, limb-darkening effects increase with narrowing Na bands. Specifically, the stellar limb darkening is less in the $\mathrm{Na}$ line core compared to the surrounding continuum, producing transit light 


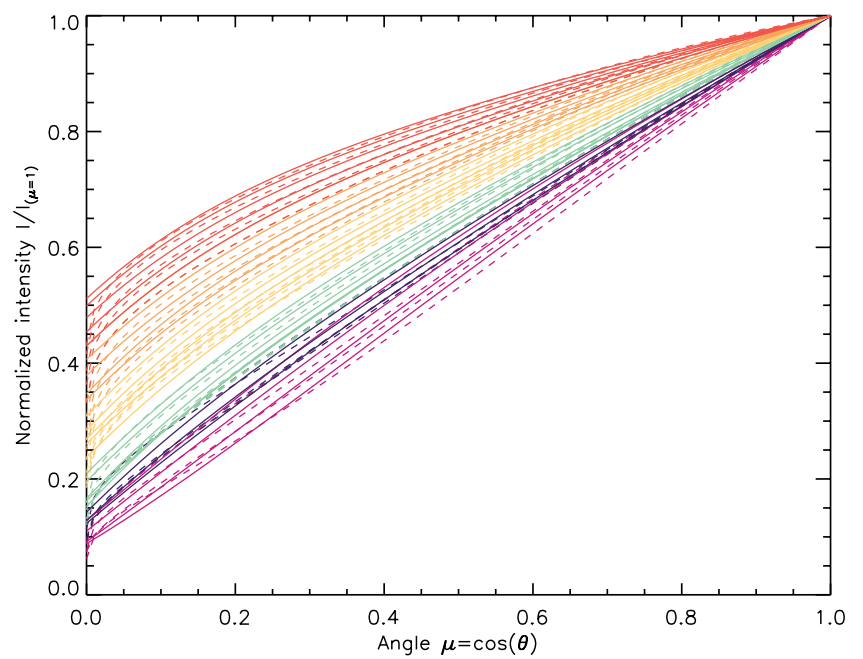

FIG. 5.-Solar limb-darkening data and ATLAS model. Plotted are 23 optical solar limb-darkening measurements from NL94 (solid lines) along with those of an ATLAS solar model (dashed lines). The wavelengths of each curve range from 3204.68 (bottom purple curve) to $10989.5 \AA$ (top red curve).

curves which are slightly more "box-shaped," with a slightly smaller depth at midtransit. When comparing the transit light curves of narrow regions centered on the $\mathrm{Na}$ line to that of a surrounding continuum, with the intention of measuring a difference in apparent planetary radius, the overall effect is to inherently underestimate the signature. Using the G750M spectra, we measured the magnitude of this effect using the stellar absorption lines ( $\mathrm{Fe}$ I, $\mathrm{Ca}$ I, Si I, etc.) and the continuum. No other large planetary atmospheric signature other than $\mathrm{Na}$ was detected in the stellar lines of the G750M data (Charbonneau et al. 2002), making them excellent probes of the effects of limb darkening independent of differing planetary radii. We produced two separate photometric light curves, one that contained only the flux from the stellar continuum and one that contained only the flux from the stellar absorption lines. The Na region was not included in either photometry. We then normalized the two curves by their out-of-transit flux and took their difference. The average intransit difference was measured, as in the differential $\mathrm{Na}$ analysis, to be $-0.0044 \% \pm 0.0015 \%$. Using the model atmosphere and calculating theoretical transits for the selected wavelengths, we found a theoretical difference of $-0.0038 \%$, which matches the observed value. This confirms that (1) the limb darkening in the stellar lines is smaller than the continuum, (2) the effect is to reduce a planetary absorption signature when measuring within a stellar line and comparing to a nearby continuum, (3) the effect is small for a typical stellar absorption line (approximately $-0.004 \%$ ), and (4) the strength of the effect is consistent with theoretical one-dimensional stellar atmosphere predictions. Although the one-dimensional stellar model used in our analysis does not contain a stellar chromosphere and assumes local thermodynamic equilibrium, the similarity between the model and data suggests that their effects are negligible on the transit light curve at these wavelengths and resolutions.

\subsection{Performance of Solar-like ATLAS Limb-darkening Models}

To gauge the performance and limitations of ATLAS atmospheric models in relation to analyzing planetary transits, we compared the limb-darkening predictions of a solar ATLAS model to the measured values of the Sun. The Sun represents the only test case where sufficiently high-precision optical limb-darkening data

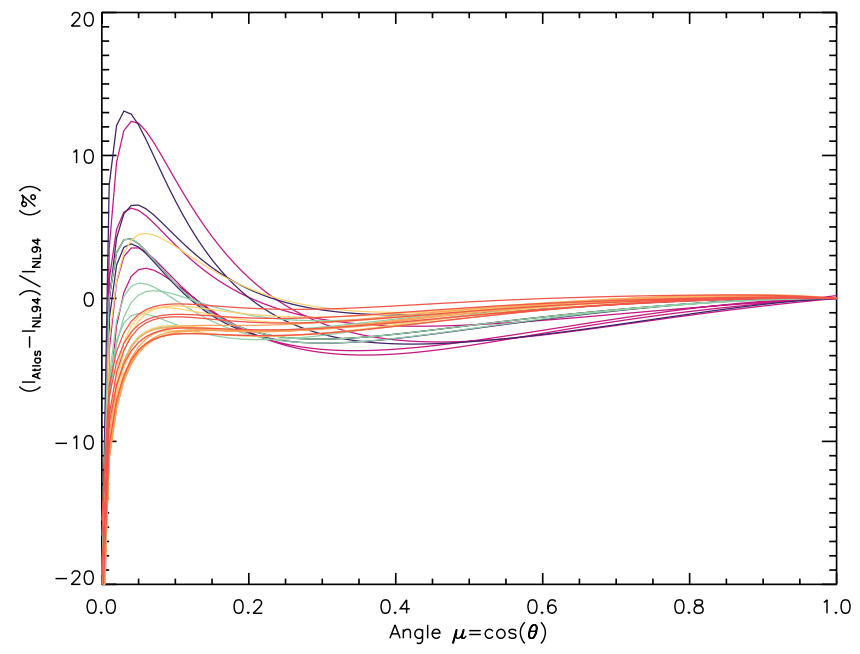

FIG. 6.-Difference between solar limb-darkening data and ATLAS model. Plotted is the difference between the limb-darkening curves predicted by the ATLAS model and measured by NL94. For $\mu$ values between $\sim 0.2$ and 1.0 , the model tends to overestimate the strength of limb darkening by a few percent. The greatest difference is observed to be at the very limb, where the models are known to have their greatest uncertainty.

exist. HD 209458 is a solar-like star classified as a G0 V, while the Sun has a similar spectral type and is a G2 V. In analyzing the performance of model atmospheres, Bertone et al. (2004) found that the ATLAS models provide a good-fitting accuracy for the physical parameters, namely, $T_{\text {eff }}$ and $\log g$, for early-type mainsequence stars, B to F, down to late-G/early-K stars. For late-K and $\mathrm{M}$ stars, the models produce systematically poorer fits, due largely to the incomplete treatment of molecular opacity, which becomes important in cooler stars. As HD 209458 is slightly warmer than the Sun, molecular opacity should correspondingly play a slightly weaker role, and the performance of the ATLAS model itself should be similar for such main-sequence stars. Testing a solar ATLAS model versus solar data represents a test of the model itself and the assumptions within the model, as the physical parameters of the Sun, which are input into the atmospheric model, are both accurately and precisely measured. The uncertainty in the measured stellar parameters themselves, and its effect on limb-darkened, corrected transit light curves, is a separate issue dealt with in $\S 4.1$.

Figure 5 shows high-precision solar limb-darkening measurements for 23 precisely chosen continuum wavelengths between 3000 and $11000 \AA$ from Neckel \& Labs (1994; hereafter NL94) and the corresponding solar ATLAS predictions, taken from the nonlinear solar ATLAS monochromatic coefficients computed by Claret (2000). The ATLAS model was calculated at a low resolution, so those wavelengths that matched closest were chosen. The ATLAS model performs well over $\mu$ values between $\sim 0.2$ and 1.0, slightly overestimating the strength of limb darkening consistently at every wavelength by a couple of percent (see Fig. 6). Our ATLAS solar comparison agrees with earlier studies (Castelli et al. 1997). The general overestimation by ATLAS models is expected, due to the inherent limitations of one-dimensional models when describing purely three-dimensional turbulent effects. This overestimation has been observed in both an F5 V and a K1 V star when comparing ATLAS models and threedimensional hydrodynamical models to high-precision interferometric measurements (Aufdenberg et al. 2005; Bigot et al. 2006). The largest differences between the model and data exist at the very limb, where ATLAS models predict a dramatic increase 


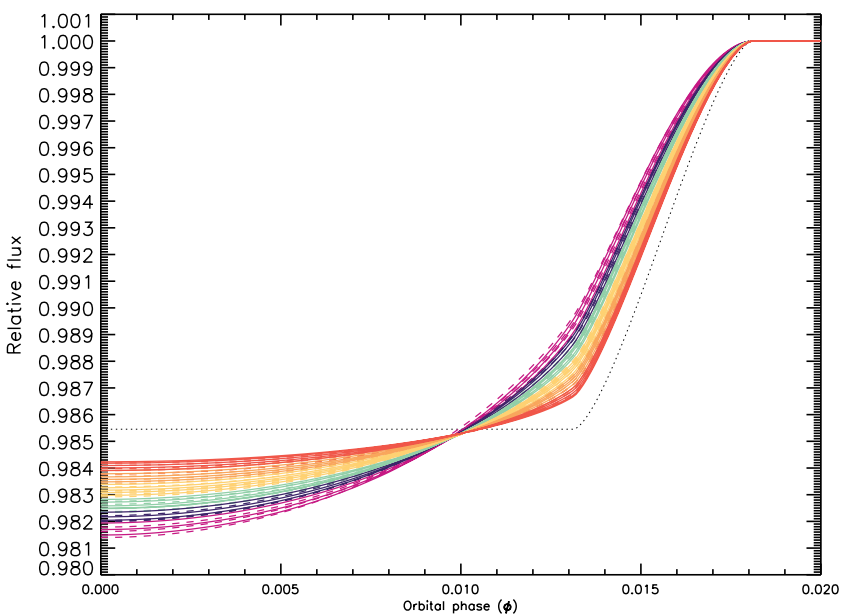

FIG. 7.- Simulated solar transits. Plotted are the simulated transits using the best-fit transit parameters of HD 209458, along with the solar limb darkening measured by NL94 (solid lines) and a solar ATLAS model (dashed lines). The dotted line represents the theoretical transit without limb-darkening effects.

in limb-darkening strength, a trend not observed in the solar limbdarkening data.

\subsection{Simulated Solar Transits}

The effect of adopting an ATLAS model to describe limb darkening in transit light curves can be seen by simulating transits with the high-precision NL94 solar data and comparing them to transits using an ATLAS solar model. For this purpose, we modeled transit light curves using the best-fit parameters of the HD 209458 system and both of the nonlinear limb-darkening coefficients predicted by the ATLAS solar model, as well as the solar data (see Fig. 7). With overall stronger limb darkening, the ATLAS models produced systematically deeper transits between second and third contact and shallower light curves during ingress and egress. The largest differences between the solar and ATLAS transits occur during ingress and egress, where transit parameter fits are relatively insensitive to the planet-to-star radius contrast, $R_{\mathrm{pl}} / R_{\mathrm{star}}$, which is the desired parameter for this study. This insensitivity, combined with its small integrated flux contribution of the limb in contrast to the rest of the stellar surface, limits the overall effect of the observed large limb model deficiencies during midtransit. At midtransit, an $\sim 2 \%$ overestimation in limb darkening (corresponding to $\mu \sim 0.5$ ) translates to an extra transit flux drop of $\sim 0.01 \%$. These trends are seen to be largely reproducible, wavelength-to-wavelength, at a $\pm 0.005 \%-0.006 \%$ level. The near-UV (NUV), where the strength of limb darkening is larger, also exhibits this general overestimation trend, but with a slightly larger scatter compared to the rest of the optical.

We corrected the transit light curve, computed with the NL94 data, for limb-darkening effects as predicted by the ATLAS model. This procedure is comparable to our limb-darkening correction procedure adopted for HD 209458 b, i.e., using the predicted limb darkening from ATLAS models to correct transit light curves which are the result of the actual limb darkening of a star. These corrected light curves can be seen in Figure 8 (top curves), where the limb-darkening overestimation by the ATLAS models translates into a slightly lower flux ratio at midtransit and slightly higher flux ratio just before third contact or just after second contact, with this general trend seen at all wavelengths. Thus, introducing the ATLAS model produces two

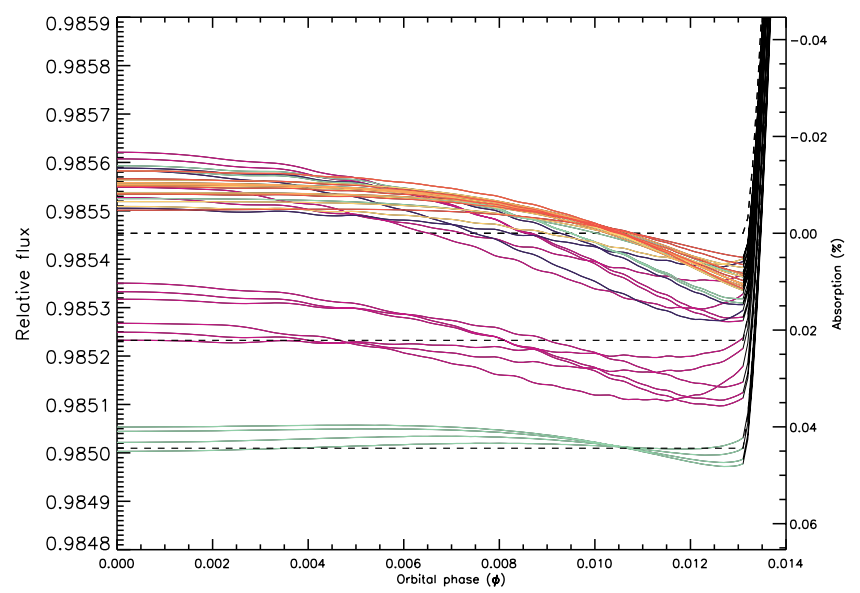

FIG. 8.- Simulated solar transits corrected by ATLAS limb darkening. Plotted are the transits generated with NL94 limb-darkening data and corrected with a solar ATLAS model. Those simulated transits containing the best-fit planetary radius $\left(1.32 R_{\mathrm{Jup}}\right.$; corresponds to $0 \%$ absorption) are plotted (top). Also plotted are simulated transits with a NUV-like absorption signature (middle, purple; calculated with a planetary radius of $1.33 R_{\text {Jup }}$ ) and simulated Na-like signature (bottom, green; calculated with $1.34 R_{\text {Jup }}$ ). The dotted lines represent the theoretical transits for all three radii in the absence of limb-darkening effects.

systematic effects: (1) a wavelength-to-wavelength flux scatter of up to $\pm 0.005 \%-0.006 \%$ and (2) a phase-dependent trend of overcorrecting the transit flux during the middle approximately two-thirds of in-transit times and undercorrecting during the remaining one-third. The first systematic effect is likely due to the internal accuracy of the ATLAS models, where uncertainties in opacity database tables and metal line blanketing, for instance, could reveal themselves. The second systematic effect is clearly related to the general trend of ATLAS models overpredicting the strength of limb darkening at a similar level in all wavelengths and tied to the treatment of convection in one-dimensional atmospheric models.

To test whether the broadband NUV and Na absorption features seen in our data could successfully be recovered using ATLAS model limb-darkening corrections, we also simulated transits using the NL94 data, which contained an extra $0.01 R_{\text {Jup }}$ in planetary radii for the NUV and an extra $0.02 R_{\text {Jup }}$ in planetary radii for selected Na-like wavelengths. These simulated absorption transit light curves were then corrected with those generated with solar ATLAS models and the best-fit planetary radius (see Fig. 8). The two simulated absorptions can easily be identified above that of the zero-absorption case, with the general phase-dependent trend largely preserved. Therefore, when averaging over a series of in-transit spectra and comparing absorption values at different wavelengths, the values of the relative absorption differences will largely be retained after the ATLAS corrections, but the flux level itself could change slightly, depending on the phase sampling (effect 2). At the extremes, using only data at midtransit, second contact, or third contact, this absolute flux difference is seen to be up to $\sim 0.01 \%$. These trends are observed in our absorption simulations. When we average over the in-transit phases and recover the input absorption signal (see Fig. 9), we get relative absorption values (above the ATLAS-corrected zeroabsorption case) of $0.026 \% \pm 0.003 \%$ and $0.046 \% \pm 0.002 \%$ for the NUV-like and Na-like signatures, respectively. These values closely match the input $0.022 \%$ and $0.044 \%$ values, indicating that ATLAS model corrections can retain relative absorption signals at a level of around $\pm 0.003 \%$. Selecting subsets which contained only similar orbital phases produced equivalent 


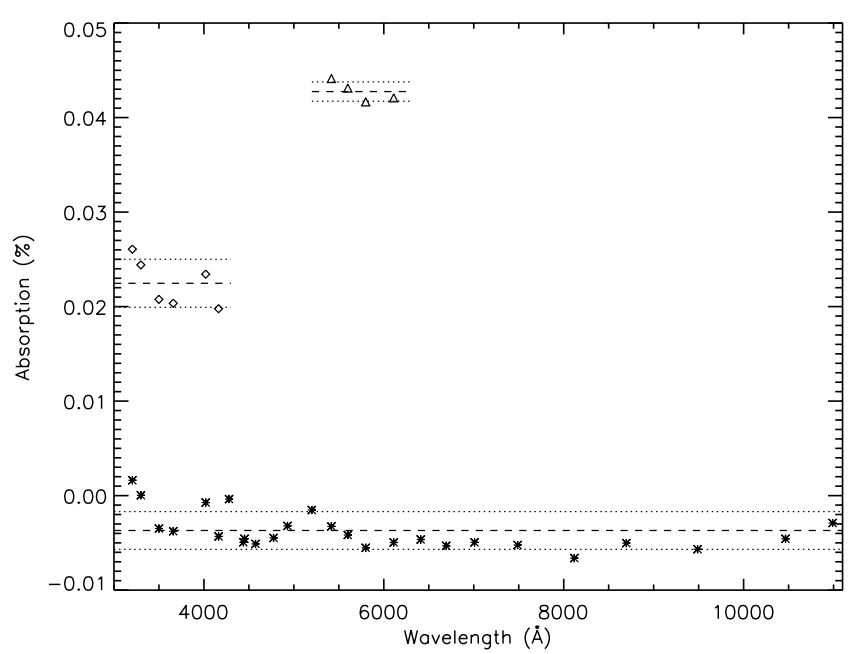

FIG. 9.- Simulated solar transit ratio spectra with NUV-like and Na-like signatures. Plotted are the average in-transit values from the ATLAS-corrected simulated solar transits (Fig. 8), which originally contained zero absorption (asterisks), a $0.0022 \%$ NUV-like signature (diamonds), and a $0.0044 \%$ Na-like signature (triangles). The two simulated differential absorption signatures are easily recovered. For each set, the average and $1 \sigma$ standard deviation are also plotted (dashed and dotted lines).

differential results, indicating that effect 2 has a limited impact on relative absorption measurements.

\subsection{Application of Solar Test to HD 209458}

The residual G430L and G750L ATLAS-fit light curves (in addition, see Fig. 4 of Knutson et al. 2007) show the same trends as observed in our solar test. Namely, the HD 209458 transit light curves show a systematically slightly higher residual flux at midtransit compared to second and third contact at an $\sim 0.01 \%$ $0.02 \%$ flux level, as in our solar test, with this trend repeated at all optical wavelengths. This indicates that the performance of the ATLAS models is similar for both HD 209458 and the Sun. When fitting for the planetary radius, in the case of HD 209458, adopting an ATLAS limb-darkening model would therefore seem to systematically fit a slightly smaller $R_{\mathrm{pl}} / R_{\mathrm{star}}$ ratio at every wavelength and thus result in smaller planetary radii (given uniform and complete phase coverage). However, this systematic error is much smaller than the uncertainty due to the stellar mass and has a negligible effect on the final determined planetary radius. This is reflected in the analysis of Knutson et al. (2007), who find planetary radii values that differ by less than $1 \sigma$ when using either the method of adopting ATLAS models or fitting for limb-darkening coefficients.

Our analysis shows that, over the entire optical regime 3000$10000 \AA$, it is possible to use ATLAS models to accurately measure the relative value of planetary radii to around a $0.005 \%$ $0.006 \%$ precision level in solar-type stars. This precision level is comparable to our highest $\mathrm{S} / \mathrm{N}$ achieved when averaging over large wavelength ranges $(>1000 \AA)$ and multiple exposures (see $\S 4.2$ ). Thus, the systematic errors introduced by assuming ATLAS models are negligible for our purposes of probing relative transit depths for signals $>0.01 \%$. The success of using ATLAS models in analyzing transits of the K0 V star HD 189733 (Pont et al. 2008) further highlights their capabilities. As a relatively cooler $\mathrm{K}$ star, molecular opacity plays a correspondingly larger role in HD 189733 than the Sun or HD 209458, yet an ATLAS model provides superior fits compared to leaving the limb-darkening coefficients as free parameters in transit fits (F. Pont 2008, private communication). As more high-precision measurements become available across a wider wavelength range, care will have to be taken when comparing transit parameters determined by separate analyses, which make different limb-darkening assumptions.

\section{ANALYSIS}

\subsection{Stellar Parameter Uncertainties}

Uncertainty in the stellar parameters $\left(T_{\text {eff }}, \log g, \log _{10}[\mathrm{M} / \mathrm{H}]\right.$, $v_{\text {turb }}$ ) can lead to a range of possible stellar models and thus a range of predicted limb darkening. As limb darkening derives mainly from intensity differences between blackbodies at different temperatures, changes in the $T_{\text {eff }}$ of the star have the largest effect on the calculated limb darkening. For HD 209458, the range of $T_{\text {eff }}$ found in the literature is from $\sim 6000$ to $6100 \mathrm{~K}$ $(6030 \pm 140 \mathrm{~K}$, Allende Prieto \& Lambert 1999; $6000 \pm 50 \mathrm{~K}$, Mazeh et al. 2000; $6117 \pm 26 \mathrm{~K}$, Santos et al. 2004; $6099 \mathrm{~K}$, Fischer \& Valenti 2005), and we have adopted a $T_{\text {eff }}$ of 6100 for this study.

We estimate the uncertainty of limb darkening inferred from stellar parameter uncertainty by estimating how large a temperature increase or decrease is necessary to produce a difference flux of $0.02 \%$ at midtransit between bandpasses, which is near the absorption signal detected in the NUV region. In our wavelength regime, the flux at midtransit in the NUV is the most sensitive to changes in limb darkening, making this test an upper limit to the $T_{\text {eff }}$ change needed at longer wavelength bandpasses and other phases. From Claret (2000), we use nonlinear limb darkening for the photometric bandpasses $U$ and $B$ calculated at three different temperatures and measure the transit depth that results. The $U$ and $B$ wavelength ranges roughly correspond to the $\sim 0.03 \%$ flux difference observed in our transmission spectrum between the NUV (3000-3700 $\AA$ ) and nearby optical (4000$5500 \AA$ ). . Using the best-fit HD 209458 parameters, we calculate that a $T_{\text {eff }}$ change from 6000 to $5500 \mathrm{~K}$ changes the $U$ band midtransit depth by $0.05 \%$ and the $B$ band by $0.03 \%$, resulting in an $\sim 0.02 \%$ change in relative flux depth. Likewise, changing the $T_{\text {eff }}$ from 6000 to $6500 \mathrm{~K}$ changes the midtransit depth by $0.05 \%$ in the $U$ band and $0.03 \%$ in the $B$ band, also resulting in an $\sim 0.02 \%$ change. Thus, an uncertainty of only $\pm 50 \mathrm{~K}$ for the $T_{\mathrm{eff}}$ of HD 209458 corresponds to a NUV limb-darkening uncertainty of only $\sim 0.002 \%$, a level well below our $\mathrm{S} / \mathrm{N}$ level.

\subsection{Red Noise}

We quantify the level of systematic "red noise" in our transmission spectrum following the procedures detailed in Pont et al. (2006). In the absence of red noise, averaging over successive measurements increases the $\mathrm{S} / \mathrm{N}$ in a predictable manner, with the standard deviation of the average varying inversely with the square root of the number of measurements, $N_{t}$. In the presence of red noise, however, the standard deviation does not decrease as quickly when averaging over a larger number of measurements, and in the limit of completely correlated noise, it becomes a constant of $N_{t}$.

To estimate the level of correlated noise in our data, we take the out-of-transit spectra, binned over $N_{w}$ pixels in wavelength, and calculate the variance and standard deviation from a distribution of points binned by $N_{t}$ successive measurements. This is repeated for an increasingly larger number of $N_{t}$ and $N_{w}$ bin sizes. We check different binning combinations to ensure that correlated noise does not appear at our binning frequency. Plotted in Figure 10 is a sample of red noise estimation from the G750L data in the $6200 \AA$ region. Typically, photon noise is seen to dominate down to precision levels of $\sim 0.02 \%$, where the effects of red noise start to become visible. For most of the optical, binning 


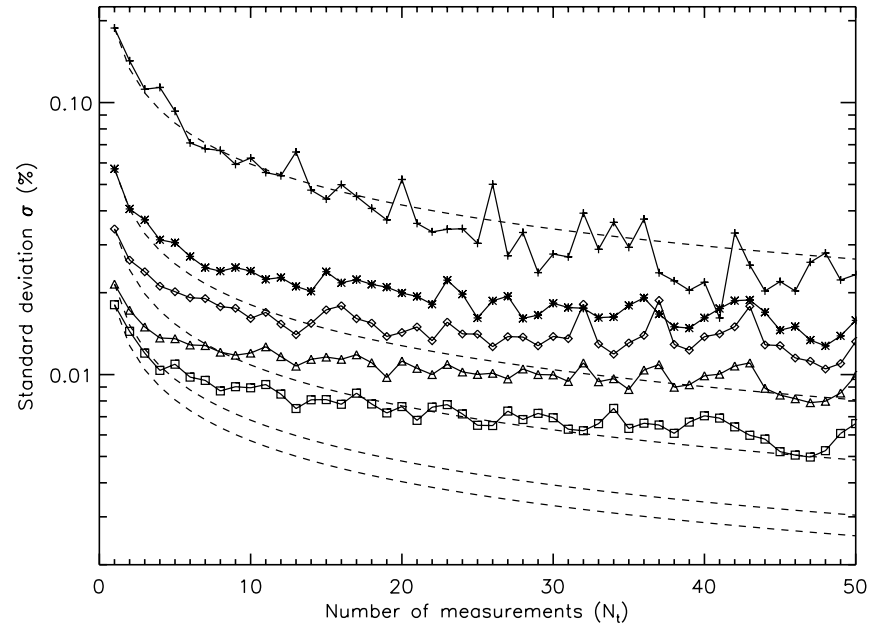

FIG. 10.- Red noise estimation. Plotted is the standard deviation of the average of $N_{t}$ successive measurements centered at $6200 \AA$, binning in wavelength by

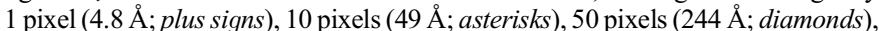
150 pixels ( $\sim 732 \AA$; triangles), and 200 pixels (977 $\AA$; squares). The five dashed lines show an uncorrelated $\sigma N_{t}^{-1 / 2}$ relation. A single pixel is dominated by photon noise and follows an uncorrelated relation. Correlated noise appears when binning by wavelength pushes $\sigma$ below $\sim 0.02 \%$. In this case, we are able to reach precision levels of $\sim 0.006 \%(\mathrm{~S} / \mathrm{N} \sim 16,000)$ when binning by $>30$ measurements over $\sim 1000 \AA$ where we are dominated by residual correlated noise resulting from systematic errors.

over large wavelength regions and multiple exposures allows us to reach precision levels of $\sim 0.006 \%$, although after binning beyond $\sim 30$ measurements, the $\mathrm{S} / \mathrm{N}$ usually does not significantly increase.

As binning over larger wavelength bands has diminishing returns, the presence of red noise gives our transmission spectra a $\mathrm{S} / \mathrm{N}$ level at moderate wavelength bins similar to that at larger wavelength bins. This is reflected in Figure 11, where all five $1 \sigma$ error bars calculated to show the significance of observed broadband features are similar to each other, although they differ in wavelength breadth. The broadband errors are also similar to the typical $1 \sigma$ uncertainty levels when binning over 16 pixels, $0.011 \%$.

\subsection{Broadband Absorption Signatures and Optical Transit Transmission Spectra}

Using the G750M and G750L spectra, we used a limb-darkened corrected transit spectral ratio to probe the full wavelength dependence of atmospheric absorption features, producing an optical transmission spectra. The relative planetary radii, when comparing between different wavelengths, are largely independent of the uncertainties of the stellar parameters and can be determined much more precisely than the actual radius values themselves (Knutson et al. 2007). Putting the low-resolution G750L data together with the G430L data (Ballester et al. 2007) allows a probe of the region from 3000 to $10000 \AA$, with the $\mathrm{Na} \mathrm{D}$ lines located in a high-S/ $\mathrm{N}$ region in the middle.

Our low-resolution transmission spectra were produced by interpolating all the systematic error-corrected spectra onto a common wavelength scale. We then produced both an average out-of-transit spectrum and an average limb-darkened corrected in-transit spectrum (using phases between second and third contact), with the ratio of the two providing our ratio spectrum. The error was computed by taking into account both photon noise and red noise, as outlined in $\S 4.2$. The G750L spectral region blueward of $5548 \AA$ at the edge of the spectrum was omitted, as

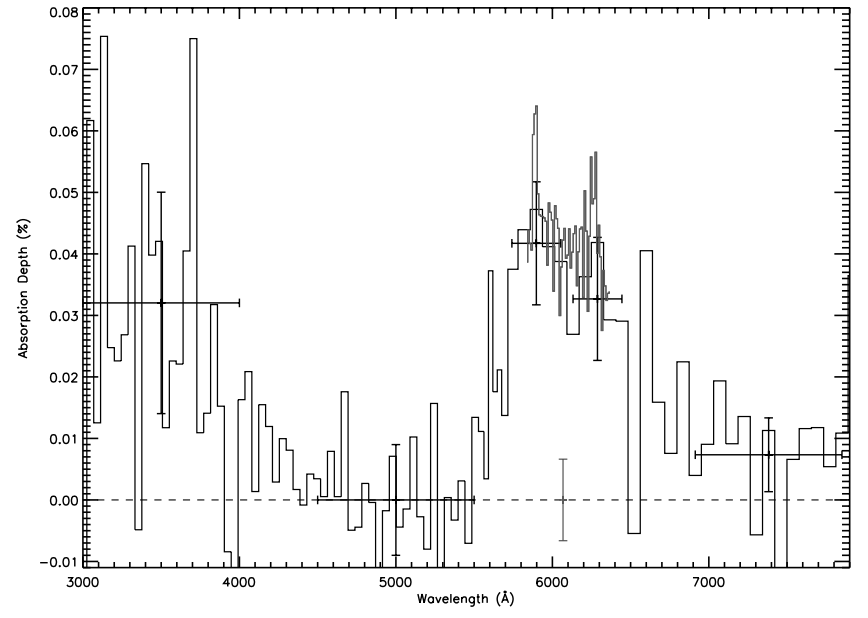

FIG. 11.-Optical transit transmission spectra of HD 209458b. Plotted is the transmission spectrum derived from the low-resolution G430L and G750L gratings (thin black line; binned over 16 pixels), as well as the medium-resolution G750M ( gray line; binned over 18 pixels, $\sim 10 \AA$ ). Also plotted are $1 \sigma$ uncertainty levels ( $y$-axis error bars) on low-resolution broadband features averaged over selected wavelength regions ( $x$-axis error bars) and a representative error bar for the binned medium-resolution data ( gray error bar). The absorption scale has been normalized to the value at $5000 \AA$ (1.444\%). The uncertainty levels include the effects of red noise.

it contains a region of very rapidly decreasing response whose spectral ratio value is $0.03 \%$ lower when compared to the higher $\mathrm{S} / \mathrm{N}$ overlapping the G430L region. This omitted G750L region is seen to have a larger amount of red noise, limiting the accuracy levels in the region to $\sim 0.02 \%-0.03 \%$, which likely explains the flux difference between the two data sets. We used the best-fit value of $1-\left(R_{\mathrm{pl}} / R_{\mathrm{star}}\right) \sim 1.455 \%$ from Knutson et al. (2007) as our baseline transit depth reference, subtracting our spectral ratio from that value to construct a plot of the atmospheric absorption profile (Fig. 11). We also performed the same procedure on the G750M data, although a shift of +0.00023 was needed to generate an average absorption level consistent with that of the lowresolution data. As the medium-resolution data were taken $3 \mathrm{yr}$ before the low-resolution data, long-term differences in starspots, for instance, could easily account for the small measured difference in absolute absorption level. The effects of the uncertainties in the transit parameters were tested by varying the period, inclination, stellar radius, and stellar mass by $1 \sigma$ (as reported in Knutson et al. 2007) and producing a new transmission spectrum. Due to limb darkening, changing the inclination has a larger effect than the other parameters, although all produce negligible effects on the final transmission spectrum. For instance, even for the NUV at $3000 \AA$, which contains strong limb darkening, changing the inclination by $1 \sigma$ still only has a $0.002 \%$ effect on the final transmission spectrum.

The wavelength range of the G750L grating extends in the red past $1 \mu \mathrm{m}$, although with significant detector fringing (Knutson et al. 2007). The data reduction procedures used here were insufficient to correct for the large fringing effect on a pixel-by-pixel basis. Consequently, those regions which showed signs of significant fringing were left out of this analysis, corresponding to wavelength regions longer than $\sim 8000 \AA$.

\section{DISCUSSION AND CONCLUSIONS}

Our optical transit transmission spectrum combining all three gratings (see Fig. 11) gives a consistent and more complete view of the observed absorption features present in HD 209458b. The low-resolution transmission spectrum reveals three prominent 
broadband absorption features: (1) a NUV absorption below $\sim 4000 \AA$, (2) a strong broadband Na feature, and (3) an additional absorption feature at $\sim 6250 \AA$. The medium-resolution data (1) show the strong Na core absorption $(\sim 0.065 \%$ in a $4.4 \AA$ line-core band) and (2) confirm the presence of the absorption feature at $\sim 6250 \AA$. . The NUV absorption region was first reported by Ballester et al. (2007) and is interpreted, along with wavelengths shorter than $5000 \AA$ by Lecavelier des Etangs et al. (2008), to be Rayleigh scattering by $\mathrm{H}_{2}$, while the long-wavelength continuum redward of $\sim 6200 \AA$ can be explained by absorption by TiO and VO (Désert et al. 2008).

Seen at low resolution, the wide Na wings and additional absorption at $6250 \AA$ place the medium-resolution data on an apparent plateau, $\sim 0.045 \%$ above that of the minimum absorption levels seen around $5000 \AA$ and redward of $7000 \AA$. As such, the total Na core absorption reaches to $\sim 0.11 \%$ within a $4.4 \AA$ band above the minimum level at $5000 \AA$. This strong $\mathrm{Na}$ absorption is much closer to the original cloudless predictions (Seager \& Sasselov 2000; Brown 2001; Hubbard et al. 2001), albeit with a more complicated Na line absorption profile (Sing et al. 2008). The large Na line-core absorption would seem to disfavor highaltitude clouds or haze cutting into the absorption signature, as recently seen in HD 189733 by Pont et al. (2008). The distinct transition from broad Na features at lower absorption depths to sharp, narrow features at high absorption depths, however, indicates that condensation of Na is likely in the upper atmosphere, which would deplete those higher altitudes of atomic Na (Sing et al. 2008).

The complicated Na D line shape and telluric contamination illustrate some of the difficulties in observing this atmospheric absorption signature in HD 209458b. Ground-based spectroscopic measurements, such as the Na detected in HD 189733b (Redfield et al. 2008), require air-mass corrections and are only sensitive to the $\mathrm{Na} \mathrm{D}$ line cores where the atmospheric atomic $\mathrm{Na}$ in HD 209458 b is substantially depleted. Furthermore, telluric $\mathrm{Na}$ contamination has a nonnegligible impact on the signature, further masking the small signal.

As seen here with $\mathrm{Na}$, transit spectra at multiple resolutions are needed to properly interpret complex transmission features. Data at low resolution, covering a broad wavelength range, place the overall features in context, while data at medium or high resolution are better suited to identify specific absorption features and probe planetary atmospheres over a large pressure range. Our tests show that for exoplanets with solar-type stellar hosts, such transmission spectra can be corrected for limb-darkening effects to a high precision with current atmospheric models.

Space-based observations on exoplanetary transits offer the extraordinary ability to provide exoplanetary transmission spectra at multiple resolutions, leading to detailed atmospheric composition information. As the $\mathrm{S} / \mathrm{N}$, resolution, and wavelength coverage of such spectra improve, multiple species can be detected and atmospheric properties inferred. The data quality of the spectra used here and results herein help indicate the full potential of the transit method, which can be further exploited with the impending repair of HST and future launch of the James Webb Space Telescope.

D. K. S. is supported by CNES. This work is based on observations with the NASA/ESA Hubble Space Telescope, obtained at the Space Telescope Science Institute (STScI), operated by AURA, Inc. Support for this work was provided by NASA through a grant from the STScI. We thank F. Pont, D. Ehrenreich, G. Hébrard, R. Ferlet, and F. Bouchy for discussions and insight. We also warmly thank our referee for comments and corrections.
Allende Prieto, C., \& Lambert, D. L. 1999, A\&A, 352, 555

Aufdenberg, J. P., Ludwig, H.-G., \& Kervella, P. 2005, ApJ, 633, 424

Ballester, G. E., Sing, D. K., \& Herbert, F. 2007, Nature, 445, 511

Bertone, E., Buzzoni, A., Chavez, M., \& Rodriguez-Merino, L. H. 2004, AJ, 128,829

Bigot, L., Kervella, P., Thevenin, F., \& Segransan, D. 2006, A\&A, 446, 635

Brown, T. M. 2001, ApJ, 553, 1006

Castelli, F., Gratton, R. G., \& Kurucz, R. L. 1997, A\&A, 318, 841

Charbonneau, D., Brown, T. M., Noyes, R. W., \& Gilliland, R. L. 2002, ApJ, 568,377

Claret, A. 2000, A\&A, 363, 1081

Désert, J.-M., Vidal-Madjar, A., Lecavelier des Etangs, A., Sing, D. K., Ehrenreich, D., Hébrard, G., \& Ferlet, R. 2008, A\&A, submitted

Fischer, D. A., \& Valenti, J. 2005, ApJ, 622, 1102

Horne, K. 1986, PASP, 98, 609

Hubbard, W. B., Fortney, J. J., Lunine, J. I., Burrows, A., Sudarsky, D., \& Pinto, P. 2001, ApJ, 560, 413

Knutson, H. A., Charbonneau, D., Noyes, R. W., Brown, T. M., \& Gilliland, R. L. 2007, ApJ, 655, 564

\section{REFERENCES}

Lecavelier des Etangs, A., Vidal-Madjar, A., Désert, J. M., \& Sing, D. K. 2008 , A\&A, 485, 865

Mandel, K., \& Agol, E. 2002, ApJ, 580, L171

Mazeh, T., et al. 2000, ApJ, 532, L55

Neckel, H., \& Labs, D. 1994, Sol. Phys., 153, 91 (NL94)

Pont, F., Knutson, H., Gilliland, R. L., Moutou, C., \& Charbonneau, D. 2008, MNRAS, 385, 109

Pont, F., Zucker, S., \& Queloz, D. 2006, MNRAS, 373, 231

Redfield, S., Endl, M., Cochran, W., \& Koesterke, L. 2008, ApJ, 673, L87

Santos, N. C., Israelian, G., \& Mayor, M. 2004, A\&A, 415, 1153

Seager, S., \& Sasselov, D. D. 2000, ApJ, 537, 916

Sing, D. K., Vidal-Madjar, A., Lecavelier des Etangs, A., Désert, Ballester, G., \& Ehrenreich, D. 2008, ApJ, 686, 667

Vidal-Madjar, A., Lecavelier des Etangs, A., Désert, J.-M., Ballester, G. E., Ferlet, R., Hébrard, G., \& Mayor, M. 2003, Nature, 422, 143

Vidal-Madjar, A., et al. 2004, ApJ, 604, L69 